\section{NUTRITION NOTES}

\section{Letter to the Editor}

\section{Maternal and Infant Nutrition and Health in Later Life}

Sir: In response to the article, "Maternal and Infant Nutrition and Health in Later Life" by Robert L. Jackson, ${ }^{1}$ let me say that the science of many disciplines is crystal clear in finding that breast feeding the human infant is the optimum method of nutrition. The reasons are well known, accepted and not in need of repetition.

Nutrition of the infant, however, is a highly complex problem involving not only science, but socioeconomics, politics, government, geography and culture. Promoting human lactation by ignoring this complexity may well lead to large numbers of mothers feeling guilty if they do not breast feed their infants. I do not feel that such guilt is justified since there is a lack of hard data in many areas of infant nutrition.

I am concerned about some statements in Dr. Jackson's paper. For example, "Nutritional imbalance in this period (four to six months postnatally) may have lasting effects not only on physical growth and development, but also on emotional and intellectual development." The evidence supporting this statement is scanty.

The hypothesis quoted that suckled breast milk becomes richer in fat aiding in appetite control and thus preventing obesity is not proven. ${ }^{2}$ As regards the higher content of cholesterol in human over cow's milk, it is stated, "In view of the rapid growth of the brain in early life and its high fat content, the amount and type of fat fed the infant may be important for the normal development of the central nervous system." The brain synthesizes its own lipid and is not relatively dependent on dietary supply.

Finally, it is stated, "The major source of calories, however, should continue to come from human milk until the infant is about 12 months of age." This is neither consistent with an earlier statement in the paper, nor desirable, particularly in the lesser developed countries where all the brouhaha of breast-versus-bottle has over-reached itself, losing sight of the still more important issue of weaning foods and the prevention of malnutrition.

In summary, let all disciplines and parties concerned interact in a mutually helpful way to lower infant mortality and morbidity and malnutrition among those world humans who matter most--children.

Frank Falkner, M.D., F.R.C.P. School of Public Health Community Health Programs Maternal and Child Health University of Michigan Ann Arbor, MI 48109

\section{Reply}

Dr. Jackson states that the statement in his paper represents his opinions on the basis of his interpretation of the data. He has prefaced each with the conditional verb "may".

Robert E. Olson, M.D., Ph.D. Editor

1. R.L. Jackson: Maternal and Infant Nutrition and Health in Later Life. Nutrition Reviews 37: 33-37, 1979

2. J.L. Smart: Human Milk Fat and Satiety: An Appealing Idea Reappraised. Early Human Development 2: 395-397, 1978 